\title{
Review on Japanese-German-U.S. Cooperation on Laser-Ablation Propulsion
}

\author{
Stefan Scharring*, John E. Sinko ${ }^{\dagger}$, Stephanie Karg*, Hans-Albert Eckel*, \\ Hans-Peter Röser**, Akihiro Sasoh ${ }^{\ddagger}$, Naoya Ogita ${ }^{\star}$, Noritsugu Umehara ${ }^{\S}$ \\ and Yosuke Tsukiyama ${ }^{\S}$ \\ *Institute of Technical Physics, German Aerospace Center, \\ D-70569 Stuttgart, Pfaffenwaldring 38-40, Germany \\ ${ }^{\dagger}$ The Ohio State University at Newark, 1179 University Dr., Newark, OH 43055, USA \\ ** Institute of Space Systems, University of Stuttgart, \\ D-70569 Stuttgart, Pfaffenwaldring 31, Germany \\ ¥Department of Aerospace Engineering, Nagoya University, Furo-cho, Chikusa-ku, \\ Nagoya, Aichi, Japan 464-8603 \\ $\S$ Department of Mechanical Science and Engineering, Nagoya University, Furo-cho, Chikusa-ku, \\ Nagoya, Aichi, Japan 464-8603
}

\begin{abstract}
We report on an international cooperation between Nagoya University (NU), Japan and DLR Stuttgart, Germany on scaling issues in laser ablative propulsion. Lessons learned from collaborative work in the laboratory will be summarized with respect to the comparability of experimental methods and corresponding standardization issues. With the background of previous experimental research at the University of Alabama in Huntsville (UAH), experimental work with $\mathrm{CO}_{2}$ lasers in a moderate (NU) and high (DLR) pulse energy range on laser ablation of POM is presented. Profilometry results of target surfaces are compared with fluence distributions from beam propagation modeling. Ablation from flat targets is reported with respect to energy and area scaling and compared with results from ablative propulsion employing parabolic nozzles.
\end{abstract}

Keywords: Laser ablation, Impulse measurement, Laser propulsion, Parabolic nozzle, Profilometry PACS: 42.60.Jf, 42.62.-b, 42.70.Jk, 52.25.Kn, 52.38.Dx, 52.38.Mf, 52.50.Jm, 78.20.Ci, 78.20.nb, $79.20 \mathrm{~Eb}$

\section{INTRODUCTION}

Various concepts and applications of laser ablation propulsion have been reviewed in [1]. Research groups working on this field, however, are widely spread over the world which demands for an intensive scientific exchange to promote laser ablation propulsion.

In the following we report on an international cooperation between Nagoya University (NU), Japan and DLR Stuttgart, Germany on scaling issues in laser ablative propulsion that was inspired by the second author's experimental background at different laboratories at UAH and NU, resp. Lessons learned from collaborative work in the laboratory will be summarized with respect to the comparability and standardization issues of experimental methods. 
TABLE 1. Systematic measurement uncertainties.

\begin{tabular}{lrrrr}
\hline & $\begin{array}{r}\text { Laser pulse energy } \\
\text { percent }\end{array}$ & $\begin{array}{r}\text { Ablated mass } \\
\text { mg }\end{array}$ & $\begin{array}{r}\text { Spot diameter } \\
\mathbf{m m}\end{array}$ & $\begin{array}{r}\text { Momentum } \\
\text { percent }\end{array}$ \\
\hline NU & 0.5 & 0.3 & 0.025 & 1 \\
DLR & 11.5 & 3 & 0.1 & 1 \\
\hline
\end{tabular}

\section{METHODS}

As a prerequisite for our collaborative work, the comparison of experimental methods, devices and analysis routines was a main topic in this cooperation. The main idea was that only a sound discussion on standardization issues would enable to reveal scaling issues between the different experimental setups. A brief summary of this work shall be given in the following. A more detailed description is found in [2-4].

The systematic errors of the experiments are listed in Tab. 1 and were considered in the error bars of the corresponding data plots according to

$$
\sigma_{\text {total }}=\sqrt{\left(\sigma_{\text {sys }}\right)^{2}+\left(\sigma_{\text {data }}\right)^{2}}
$$

where $\sigma_{\text {data }}$ indicates the relative data scatter of measurement set (standard deviation), $\sigma_{\text {sys }}$ denotes the relative systematic error and $\sigma_{\text {total }}$ gives the overall relative error. If several data sets were summarized in one data point, the weighted average and its standard deviation were taking into account for variations in data scatter.

\section{EXPERIMENTAL SETUP}

Our comparative measurements focused on two very different $\mathrm{CO}_{2}$ laser systems: a table-top TEA laser (by SLCR Lasertechnik $\mathrm{GmbH}$, Düren, Germany) with moderate pulse energies at Nagoya University and an electron-beam sustained high energy laser at DLR Stuttgart. The parallel work in both scientific groups was accompanied by a collaborative work phase I in Stuttgart in July 2009 and phase II in Nagoya in March 2010 .

\section{Laboratory setup Nagoya}

The experiments at Nagoya University focused on experiments in vacuum with parabolic nozzles. The $\mathrm{CO}_{2}$ laser provided pulses with energies of up to $10 \mathrm{~J}$ exhibiting a temporal profile with $90 \pm 10 n s$ FHWM spike and $1.4-1.5 \mu$ s tail (time constant). The laser beam was reflected from two molybdenum mirrors with focal length $f \approx 30 \mathrm{~m}$ and delivered through a $50 \mathrm{~mm}$ aperture and $\mathrm{ZnSe}$ window into a $\oslash 0.7 \mathrm{~m} \times 2.29 \mathrm{~m}$ vacuum chamber. Inside the chamber the beam was directed to the target by two molybdenum mirrors. The variable aperture was set to match beam and nozzle diameter. Two types of parabolic nozzle geometries (for details: type 1 and 2 in [5]) and POM target geometries 


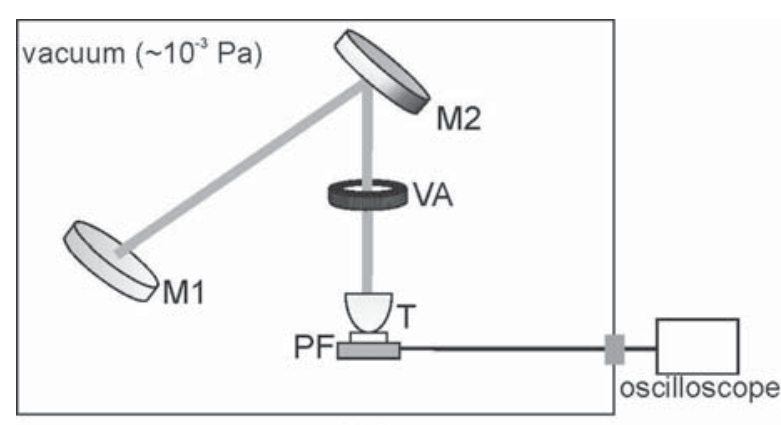

(a)

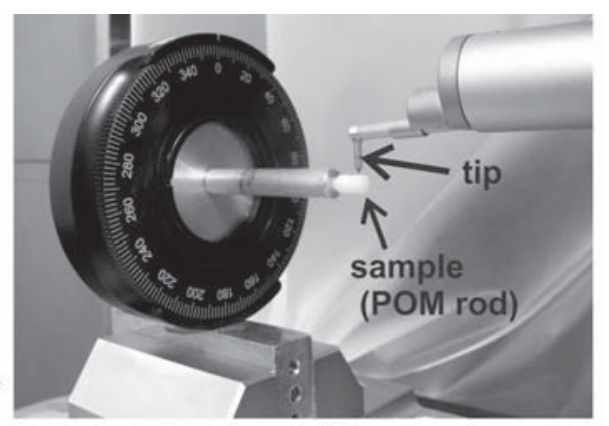

(b)

FIGURE 1. (a) Setup inside vacuum chamber; M1, M2: molybdenum mirrors, VA: variable iris aperture, T: parabolic nozzle and target, PF: piezo force sensor. (b) Profilometer (Surftest SV-3100, Mitsutoyo) and rotational mount with target.

with rod diameters $1 \mathrm{~mm}, 2 \mathrm{~mm}$ and $3 \mathrm{~mm}$ were used. Ablation experiments in ambient air and vacuum $\left(\approx 5 \times 10^{-3} \mathrm{~Pa}\right)$ were performed in the same experimental setup. For reference purposes, additional data for air breakdown were recorded. For these the POM targets were replaced by a copper ignition pin of $1 \mathrm{~mm}$ diameter [6]. Target mass and surface profiles were measured before and after ablation, usually after 5 to 10 pulses. A rotational mount allowed us to record profiles at different angular positions on the rod. For reference a small marker was placed at the 0 degree position. The imparted impulse was recorded with two different piezoelectric force sensors (PCB 208C04 and 208C01). The sensors were connected via a vacuum feed through to a signal conditioner, which passed the amplified signals to a digital oscilloscope.

\section{Laboratory setup Stuttgart}

The experiments at DLR Stuttgart were carried out at an electron-beam sustained $\mathrm{CO}_{2}$ high energy laser operating at $E_{L}=25-175 \mathrm{~J}$ delivering an average laser power of up to $10 \mathrm{~kW}$ in repetitive mode. The laser pulse exhibits a spike of $290-370 \mathrm{~ns}$ FHWM length and a tail of $7.2-10 \mu s$ which carries the main part (88 to $96 \%$ ) of the laser pulse energy [2]. The beam diameter is $d_{x}=80 \mathrm{~mm}$ and $d_{y}=79 \mathrm{~mm}$, resp., with a beam quality of $M_{x}^{2}=81$ and $M_{y}^{2}=71$, resp. Fundamental research on beamed energy propulsion focused on experiments with a parabolic nozzle (pendulum [7], wire-guided [8], free flight [9]), referred to as 'Bohn bell' [10], with a diameter of $100 \mathrm{~mm}$ (focal length $f=10 \mathrm{~mm}$ ) that was adapted to the beam diameter. The usage of a cylindrical rod of POM propellant on its symmetry axis was widely investigated [7]. Profilometric analysis of these rods at NU enabled comparison with impulse coupling data from laser ablation at flat targets with respect to fluence. Figure 2 shows the experimental setup for laser ablation experiments with flat POM targets placed in the focused laser beam (spot size $\approx 2 \mathrm{~cm}^{2}$ ).

Whereas the technical realization of suitable laser sources for the application in Earthbased launch of nanosatellites seems to be a long-term issue [11], laser sources with 


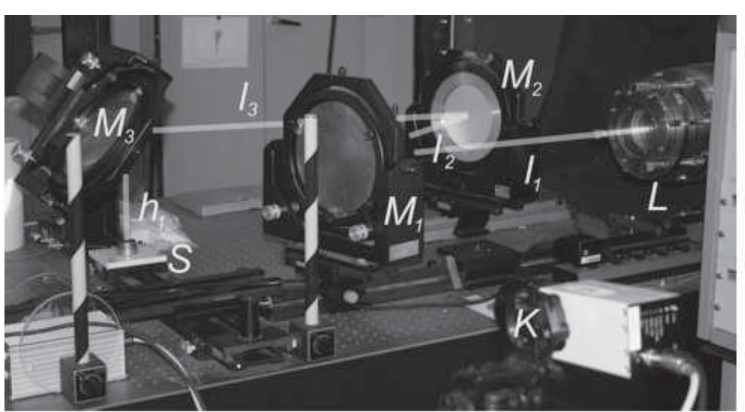

(a)

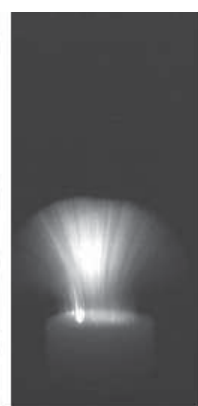

(b)

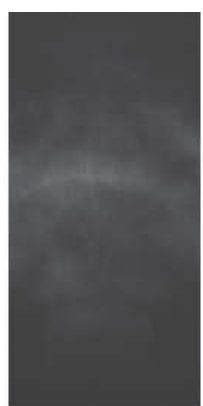

(c)

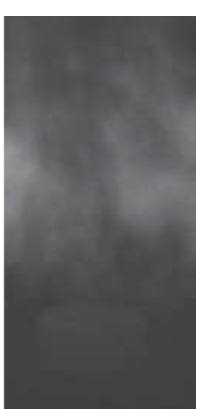

(d)

FIGURE 2. (a) Experimental setup for impulse coupling at flat POM targets at the DLR high energy laser: The laser beam from the output coupler $L$ is directed by mirror $M_{1}$ on a concave mirror $M_{2}$ with a focal length of $f=1 \mathrm{~m}$. The focused light is directed by mirror $M_{3}$ to the target that is placed on the piezoelectric sensor $S$ (PCB Piezotronics, 200C20). The ablation process is recorded by a highspeed camera $K$ (Redlake, Motion Scope M3). Three subsequent frames at $4000 \mathrm{fps}$ of an ablation event of POM copolymer at $E_{L}=83 \mathrm{~J}\left(\Phi=43 \mathrm{~J} / \mathrm{cm}^{2}\right)$ are depicted in (b) to (d).

moderate power already exist that can be used for in-space applications. Hence, scaling experiments with small parabolic nozzles $(1-3 \mathrm{~cm}$ diameter $)$ were performed at lower pulse energies $(\approx 1-20 J)$ at an air-cushion table, cf. [5], however, under atmospheric conditions. Collaborative work was intended to get a deeper insight by comparative experiments in vacuum at NU.

\section{RESULTS AND DISCUSSION}

Momentum coupling by air breakdown and / or laser ablation in parabolic nozzles has been widely investigated at DLR Stuttgart, while the work at NU in this topic focuses on momentum transfer at flat target surfaces and area scaling. The latter phenomenon is mainly characterized with respect to the incident fluence $\Phi$, while the total laser pulse energy $E_{L}$ is the usual variable for focused light in parabolic nozzles. Our main interest was whether the comparison of experimental results from both approaches would shed new light into the laser propulsion mechanism.

\section{Beam propagation modeling and profilometry of ablative targets (Nagoya - Stuttgart)}

The fluence distribution on a cylindrical propellant rod inside a parabolic reflector was modeled with a grid of 4,000 up to 9,000 rays using a raytracing software, Optica 3.0, taking into account for the laser beam divergence and fluence profile. Profilometry data on the ablation depth of the targets showed a good correlation $\left(\overline{R^{2}}=0.85\right)$ with fluence modeling results, allowing for the calculation of a threshold fluence for laser ablation of POM of $\Phi_{t}=0.44 \pm 0.06 \mathrm{~J} / \mathrm{cm}^{2}$. These findings are consistent with experimental 


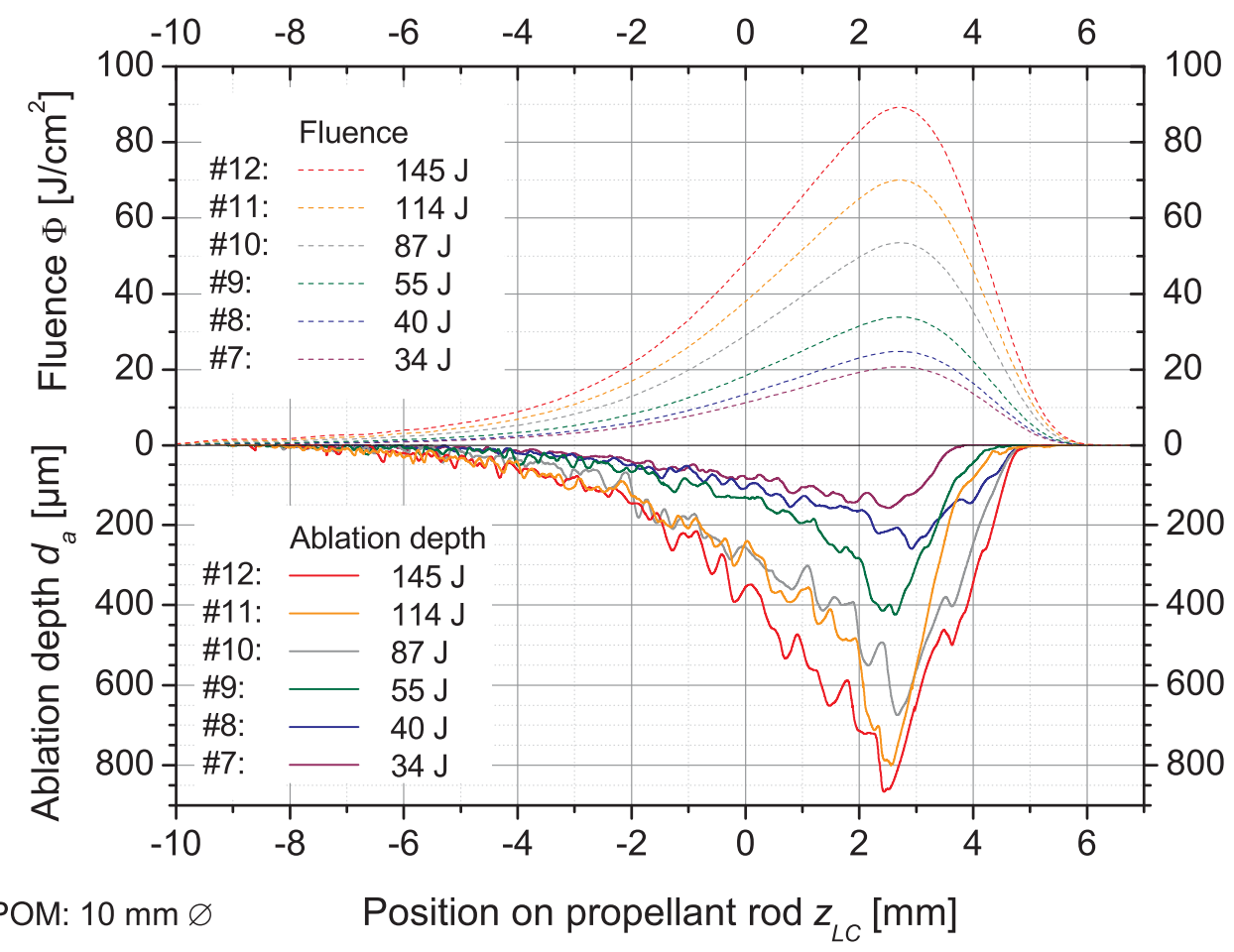

FIGURE 3. Fluence distribution and ablation profile (5 shots each) on the surface of a cylindrical POM $\operatorname{rod}(10 \mathrm{~mm} \oslash)$ inside a parabolic lightcraft.

results from flat targets [12]. In the following, we used the raytracing model to derive the (inhomogeneous) fluence on cylindrical targets from the Nagoya experiments to derive both ablation area and average fluence for comparison with flat targets.

\section{Impulse coupling of flat POM targets in ambient air (Nagoya - Stuttgart)}

At both laser systems, $c_{m}$ and $I_{s p}$ have been measured for unconfined flat targets with piezoelectric sensors for focused and unfocused laser pulses. Especially for higher fluences in the range of 10 to $100 \mathrm{~J} / \mathrm{cm}^{2}$ great discrepancies have been found in the datasets of $c_{m}(\Phi)$ for the different laser systems, cf. Fig. 4 (a). Since both laser systems differ greatly with respect to the pulse length, special care has to be taken for scaling and comparison. It is common to take into account for differences of laser systems by plotting $c_{m}$ vs. $I \cdot \lambda \cdot \sqrt{\tau}$, which traces back to the plasma regime where $c_{m}$ scales with $(I \cdot \lambda \cdot \sqrt{\tau})^{-1 / 4}[13]$. However, in Fig. 4 (b) the discrepancies between the datasets even appear more clearly, which can be attributed to the corresponding fluence threshold. According to [14], for POM the plasma regime starts at $I \cdot \lambda \cdot \sqrt{\tau} \geq 6000 \mathrm{~W} \sqrt{\mathrm{s}} / \mathrm{m}$, whereas below this threshold, the vaporization regime is located. 


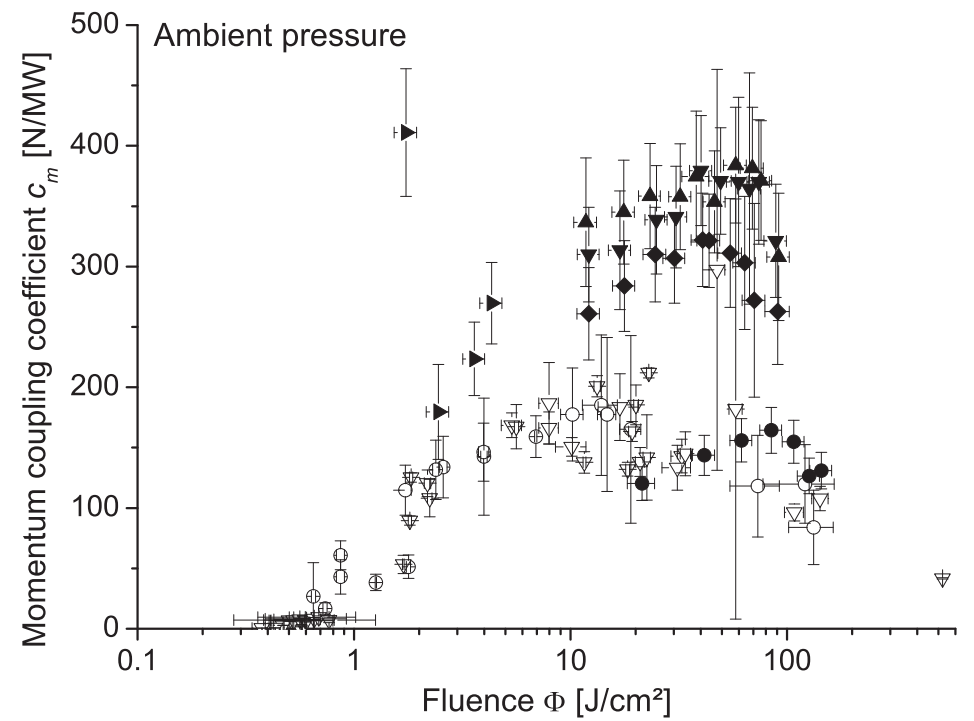

(a)

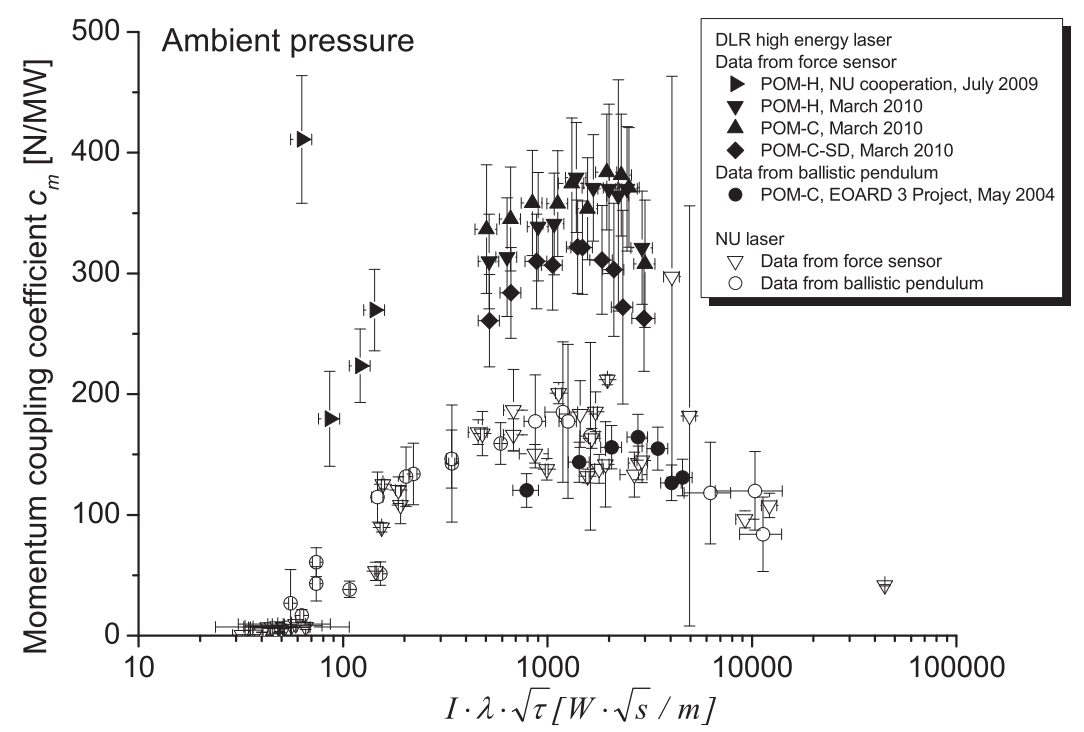

(b)

FIGURE 4. Coupling coefficient for laser ablation of POM under focused $\mathrm{CO}_{2}$ laser pulses: Data of NU and DLR from force sensor and pendulum measurements with respect to polymer modifications (homopolymer ('-H'), copolymer ('-C'), with sootless, antistatic additive ('-SD')). The legend refers to both graphs.

In the vaporization regime, however, various models exist and have been reviewed together with their approximations in [15]. They are given in the form

$$
c_{m}=f_{m} \cdot f_{v}
$$


where $f_{m}$ mirrors the ablation mechanism (photochemical, or better: following Beer's law, and photothermal) and $f_{v}$ reflects the model for the exhaust velocity (Index A: [16], Index B: [17]) yielding 4 major model combinations, denoted as 1, 5, 6, and 10 in [15], with

$$
\begin{gathered}
f_{m}=\sqrt{\frac{2 \rho \cdot \ln (\xi)}{\alpha \cdot \Phi_{t}}}(\text { Beer's law model })^{\prime} \\
f_{m}=2 \cdot \sqrt[4]{\frac{D_{T} \cdot \tau \cdot \rho^{2} \cdot \ln (\xi)}{\Phi_{t}^{2}}}(\text { Photothermal model }) \\
f_{v}=\sqrt{\frac{\xi-1-\ln (\xi)}{\xi^{2}}}(A: \text { Phipps }) \\
f_{v}=\sqrt{\frac{\xi^{2}-\xi \ln (\xi)-1}{\xi^{3}}}(B: \text { Sinko and Gregory })
\end{gathered}
$$

with

$$
\xi=\Phi / \Phi_{t}
$$

where $\Phi$ is the incident fluence, $\Phi_{t}$ the threshold fluence for ablation, $\rho$ the density of the ablated propellant, $D_{T}$ its thermal diffusivity, $\tau$ the laser pulse length, and

$$
\alpha=\frac{\alpha_{e f f}}{\tau_{m}}
$$

with the effective absorption coefficient $\alpha_{e f f}$ at high fluence and the transmissivity $\tau_{m}$ of the propellant's surface [18], and

$$
D_{T}=\frac{\lambda_{t}}{\rho \cdot C_{p}}
$$

with the thermal conductivity coefficient $\lambda_{t}$ and the specific heat capacity $C_{p}$.

The data from DLR high energy laser were compared with the presented models for the vaporization regime, as depicted in Fig. 5. With the photothermal models, the definition of the laser pulse length is crucial, and the range of $7 \mu s$ to $15 \mu s$ covers the range of minimum pulse length (FWHM) to maximum pulse length (pulse completely decayed). In general, the photothermal models fit the data much better than the photochemical models. However, $c_{m}$ is underestimated in the most cases. This may be attributed to the experimental conditions (ambient air), since the models had been developed for vacuum conditions. Combustion effects, especially at higher fluences, may enhance $c_{m}$ significantly, as reported in [7]. The boost of $c_{m}$ under ambient atmosphere compared to vacuum conditions can be estimated according to [21] from the propellant's specific combustion heat.

Since $c_{m}$ scales with $\sqrt[4]{\tau}$ for the photothermal models, it is reasonable to normalize the experimental data from both laser systems accordingly, which leads to a rather good accordance, cf. Fig. 6. 


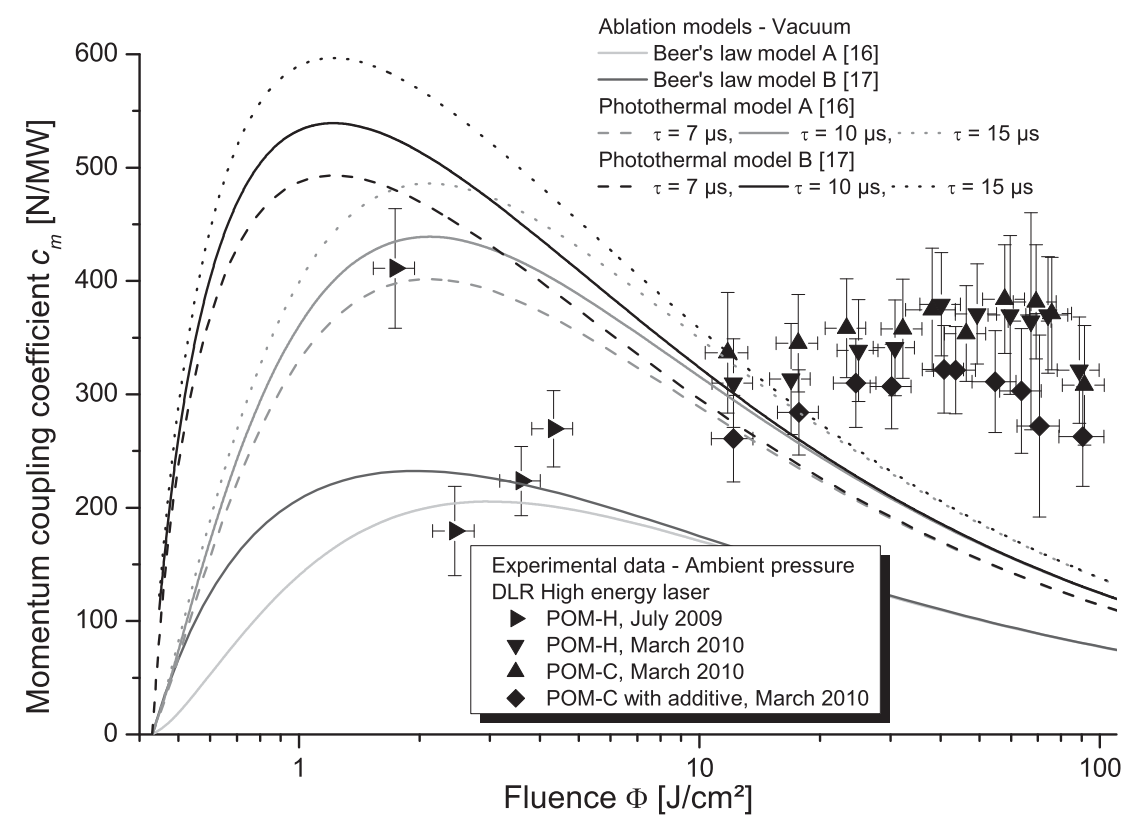

FIGURE 5. Impulse coupling data from DLR high energy laser in comparison with various model approaches for the vaporization regime $\left(\Phi_{t}=0.43 \mathrm{~J} / \mathrm{cm}^{2}[12], \alpha=2.510^{6} \mathrm{~m}^{-1}\right.$ [19], $\rho=1410 \mathrm{~kg} / \mathrm{m}^{3}$ [20], $\lambda_{t}=0.31 \mathrm{~W} / \mathrm{K} \cdot \mathrm{m}$ [20], $C_{p}=1500 \mathrm{~J} / \mathrm{kg} \cdot \mathrm{K}$ [20]). In [20], for POM-H and POM-SD slightly different values are given: $\rho=1420 \mathrm{~kg} / \mathrm{m}^{3}$ (POM-H) and $1330 \mathrm{~kg} / \mathrm{m}^{3}$ (POM-SD), $\lambda_{t}=0.30 \mathrm{~W} / \mathrm{K} \cdot \mathrm{m}(\mathrm{POM}-\mathrm{SD})$, $C_{p}$ : n.d. (POM-SD).

Furthermore, both datasets are in quite good agreement with respect to the specific impulse, cf. Fig. 7 (a). Hence, the main difference between the two laser systems can be found in the ablated mass density. With $\sigma=c_{m} \cdot \Phi=\mu \cdot\left\langle v_{e}\right\rangle$, where $\mu$ is the ablated mass areal density, $\sigma$ the area impulse density, and $\left\langle v_{e}\right\rangle$ the first moment of the exhaust velocity distribution [15], the specific impulse $I_{s p}$ can be expressed as

$$
I_{s p}=\frac{c_{m} \cdot \Phi}{g \cdot \mu}
$$

with

$$
\begin{gathered}
\mu \approx \frac{\rho}{\alpha_{e f f}} \ln \xi(\text { Beer's law model }) \\
\left.\mu \approx 2 \rho \sqrt{\tau \cdot D_{T} \cdot \ln \left(\frac{\chi \cdot \Phi}{2 \rho \cdot C_{p} \cdot T_{b} \sqrt{\pi \cdot D_{T} \tau}}\right)} \text { (Photothermal model }\right)
\end{gathered}
$$

where $\chi$ is a transmission term and $T_{b}$ is the boiling or vaporization temperature of the ablated material $[12,18]$. The experimental data from the DLR laser were compared with results from both models, as depicted in Fig. 7 (b). The results for the photothermal model with the material data as given above, $\chi=0.9$ [22], and $T_{b}=525 \mathrm{~K}$ [23] greatly exceed the experimental data. This was already observed with different datasets 


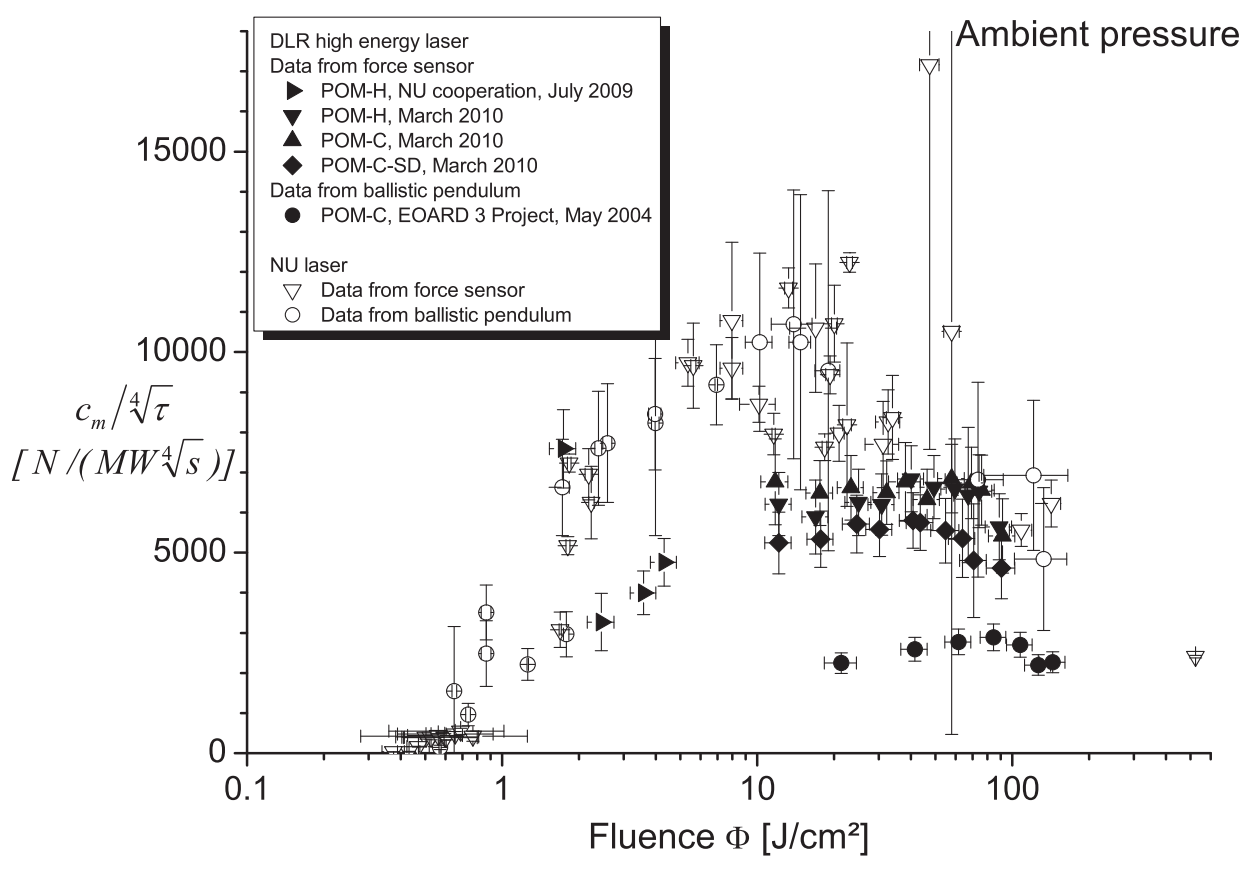

FIGURE 6. Data comparison for the different laser systems by scaling for the vaporization regime (photothermal model).

and deduced to a possible wrong estimation of the ablated mass [23]. However, the photochemical models yield fitting results that are in rather good accordance with literature data $\left(\alpha_{e f f} \cdot \tau_{m}=152 \pm 24 \mathrm{~cm}^{-1}\right.$ in [18]). These contradictory findings suggest that both photothermal and photochemical effects should be considered for the modeling of $c_{m}$ and $I_{s p}$.

\section{Nozzles and flat targets in ambient air (Nagoya - Stuttgart)}

Laser ablation in a nozzle under ambient atmosphere can be characterized with respect to various baselines. The usage of data from unconfined, flat targets has been presented for laser ablation inside conical nozzles in [18], whereas laser ablation in reflecting parabolic nozzles [7] and advanced devices [24] has often been referenced with respect to data from pure air breakdown. The latter method underlines the impact of the propellant, whereas the first one highlights nozzle effects. Both methods were applied to the data from ablation of POM inside parabolic nozzle \# 1, as depicted in Fig. 8. From Fig. 8 (a) it can be seen that within the investigated range $c_{m}$ increases with the incident fluence, whereas Fig. 8 (b) shows that for higher laser pulse energies the dependency from the rod diameter is not so pronounced. It can be seen that from around 2 to $5 \mathrm{~J}$ the larger part of the imparted momentum can be deduced to laser ablation whereas especially for energies $>8 \mathrm{~J}$ the propulsion process appears to be dominated by detonation of the air-propellant plasma. 


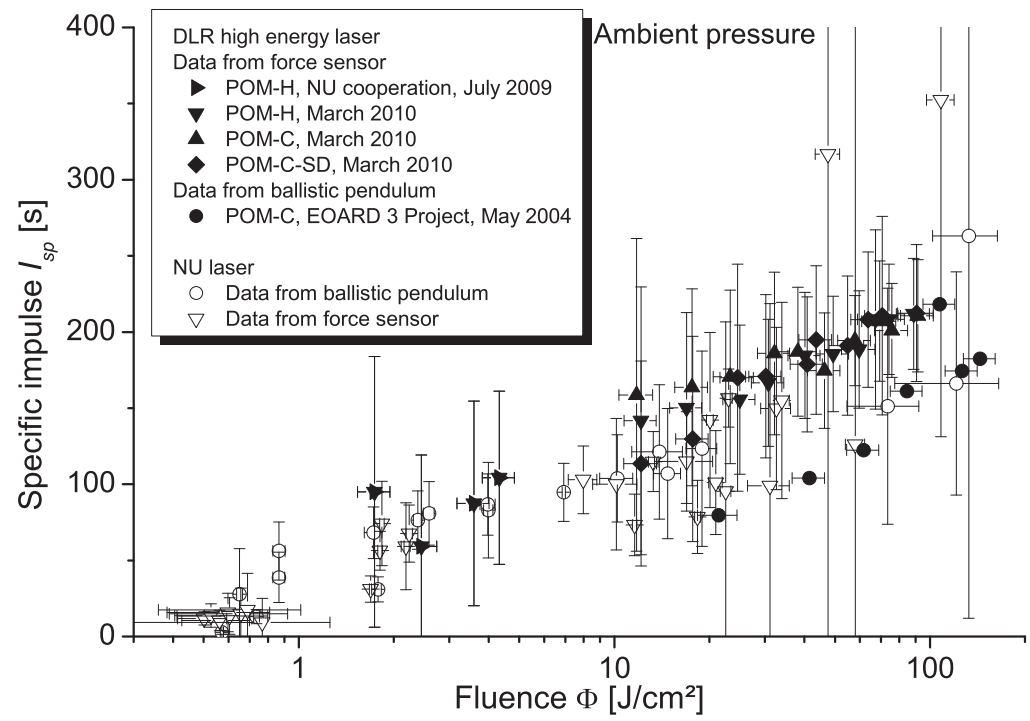

(a)

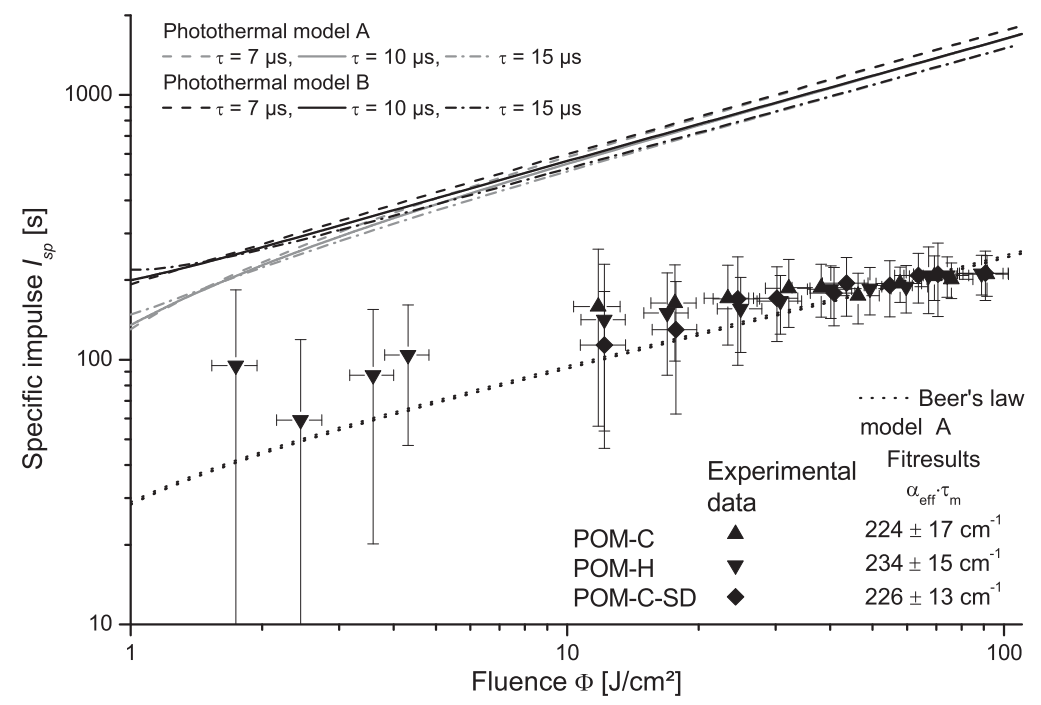

(b)

FIGURE 7. Comparison of the DLR data on specific impulse with NU data (a) and with model data (b). Photothermal models are based on material data and the range of laser pulse length at the DLR high energy laser. Datafits with photochemical model B deviate only marginally from model A fits.

\section{Nozzles and flat targets in vacuum (Nagoya)}

Confining geometries, where the target geometry or the ambient atmosphere restrict the expansion of the ablation products, can increase the interaction time between exhaust 


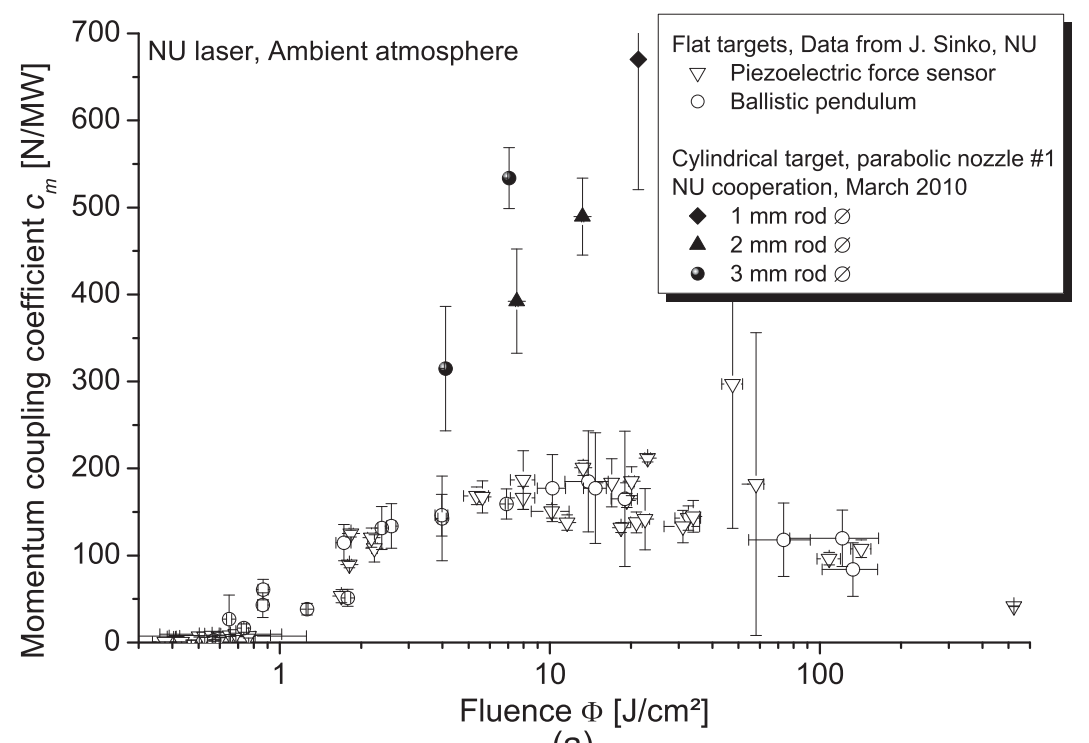

(a)

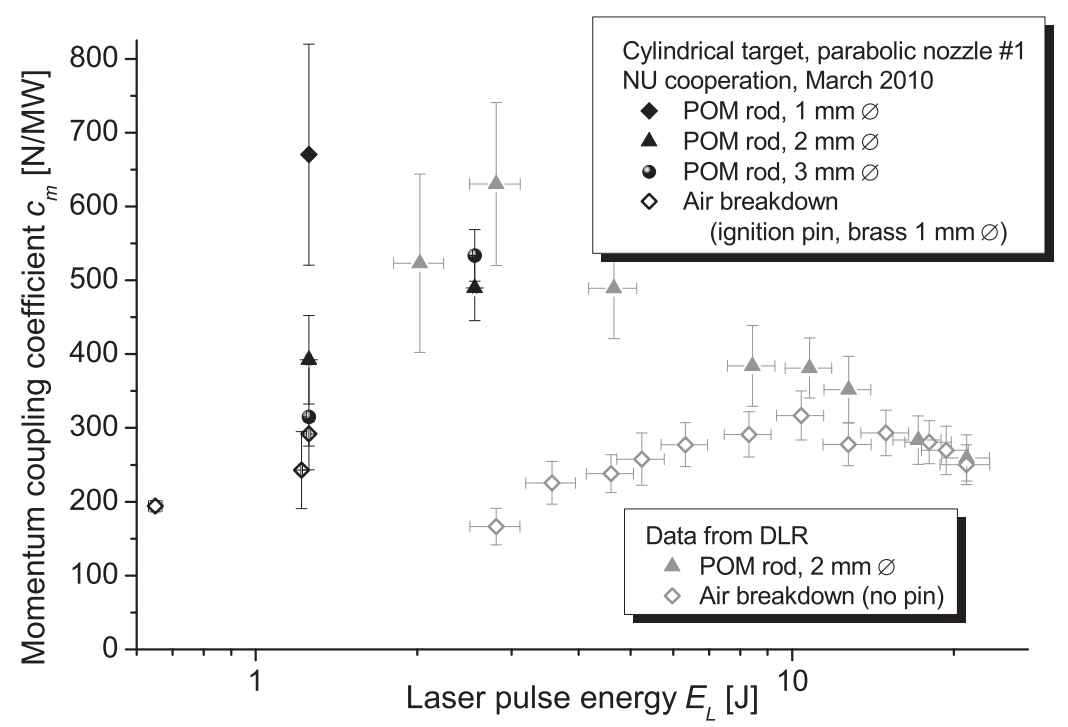

(b)

FIGURE 8. Momentum coupling by ablation of POM under ambient atmosphere in a parabolic nozzle in comparison with unconfined, flat targets (a) and pure air breakdown in a nozzle (b).

and vehicle and thus enhance the imparted momentum [14]. We compared momentum coupling of parabolic nozzles in vacuum with unconfined, flat targets, cf. Fig.9. While for both parabolic geometries the data of $c_{m}$ and $I_{s p}$ for rods with $2 \mathrm{~mm} \oslash$ match with the results found for flat targets, at high fluence ablation from POM rods of $3 \mathrm{~mm} \oslash$ yields a doubled momentum. Since the specific impulse is doubled as well, this effect is not due 


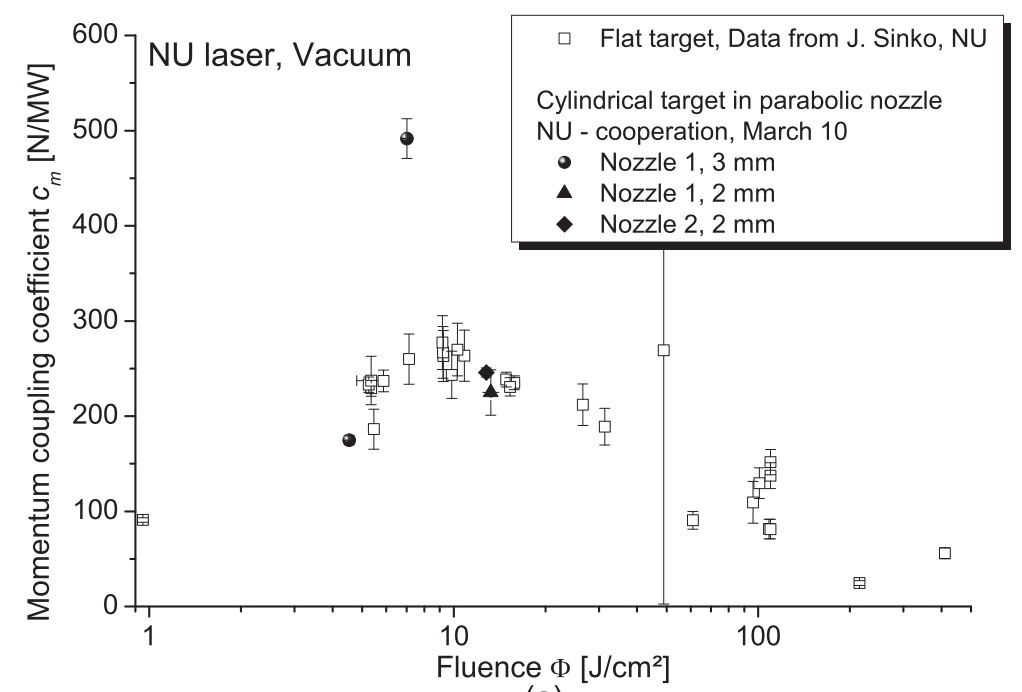

(a)

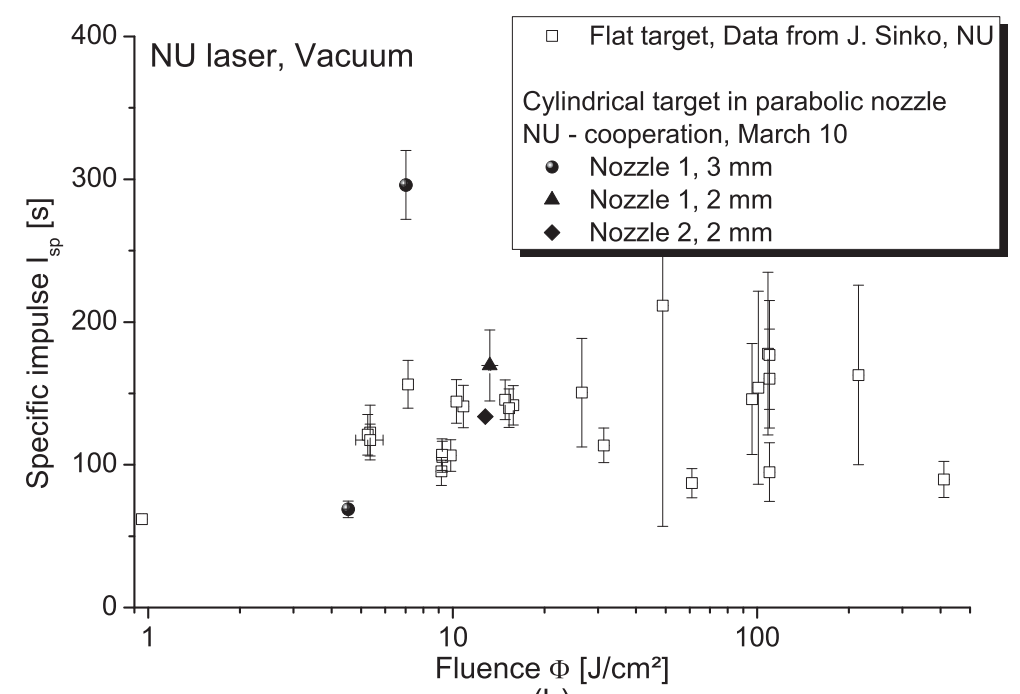

(b)

FIGURE 9. Momentum coupling (a) and specific impulse (b) for ablation of POM from cylindrical rods in parabolic nozzles and flat, unconfined targets in vacuum (pressure in the range of $1.0 \cdot 10^{-4}$ to $1.4 \cdot 10^{-3} \mathrm{~Pa}$ (nozzles) and $<5 \cdot 10^{-3}$ to $5 \cdot 10^{2} \mathrm{~Pa}$ (flat targets), resp.)

to increased ablated mass, but may be attributed to prolonged interaction time between the laser pulse and the ablation exhaust during its expansion. At fluences of around 5 to $20 \mathrm{~J} / \mathrm{cm}^{2}$ the exhaust velocity from flat targets is about 1 to $1.5 \mathrm{~mm} / \mu \mathrm{s}$. Since the free space between the ablation surface on the cylinder surface and the wall of the impulse receiver ranges from 3 to $5.5 \mathrm{~mm}$ in the experiment, the ablation jet can acquire additional laser pulse energy before its interaction with the nozzle wall $(\tau<0.5 \mu s)$. This 


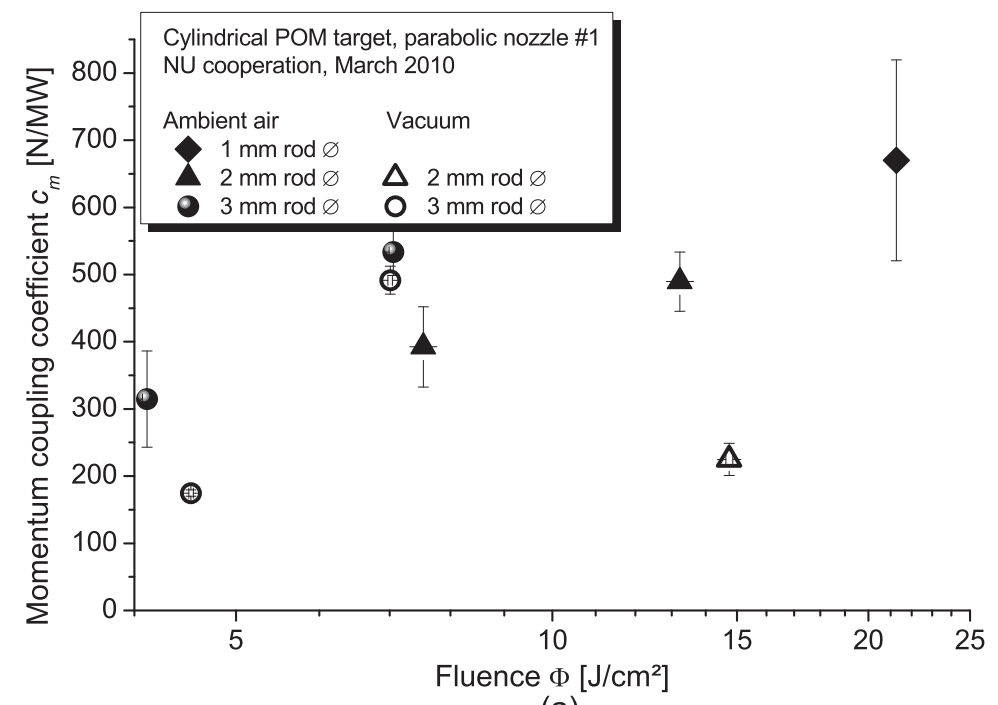

(a)

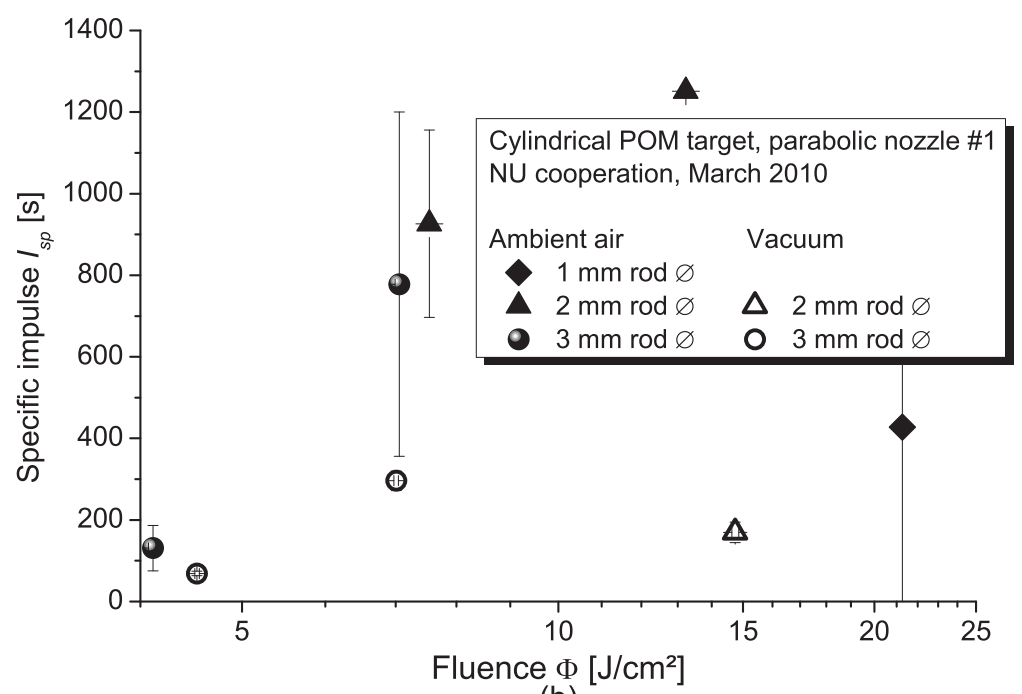

(b)

FIGURE 10. Comparison of momentum coupling (a) and specific impulse (b) vs. incident fluence on a propellant rod of POM inside a parabolic nozzle under ambient air and vacuum conditions.

feature suggests impulse enhancement by a light reflecting nozzle. However, it appears that strong dependencies on fluence as well as on the geometric configuration of ablative propellant and reflector/nozzle exist that should be subject of future research.

The results from experiments with POM rods in parabolic nozzle \# 1 in vacuum and ambient air are summarized in Fig. 10. Whereas for $d_{P O M}=2 \mathrm{~mm}$ a larger $c_{m}$ is achieved in ambient air than in vacuum, for $d_{P O M}=3 \mathrm{~mm}$ such an increase can only be stated around $4 \mathrm{~J} / \mathrm{cm}^{2}$, but not at $7 \mathrm{~J} / \mathrm{cm}^{2}$. The contribution of atmospheric air to the imparted 
momentum can be seen as well from the $I_{s p}$ data. However, these measurements have an orientating character and represent only a sparse database.

\section{SUMMARY}

In our collaboration, we experienced great benefit and inspiration from practical work together in the laboratory as well as in scientific discussions about our different measurement approaches and the specific topics we focus on in laser ablation propulsion. Two lasers with very different parameters were employed enabling for intensive studies on scaling issues in laser propulsion for a future lightcraft mission. At the same time, the different setups showed up the demand for standardization issues which were discussed in previous papers. We have shown some experimental results that link laser ablation from flat targets with ablation inside nozzles. For DLR Stuttgart, these experiments conclude our work on remotely beamed energy propulsion with parabolic nozzles building a bridge to our future experimental research on laser ablation in the field of micropropulsion.

\section{ACKNOWLEDGMENTS}

Financial support by DLR funds for International Cooperation is gratefully acknowledged. Additional support was provided through the second author by a 2009 Global Center of Excellence Young Researcher Award and by the Ohio State University of Newark. Support was also provided by Nagoya University and the Micro-Nano Division of the Global Center of Excellence. The authors also want to thank Sebastian Walther for technical support.

\section{REFERENCES}

1. C. Phipps, M. Birkan, W. Bohn, H.-A. Eckel, H. Horisawa, T. Lippert, M. Michaelis, Y. Rezunkov, A. Sasoh, W. Schall, S. Scharring, and J. Sinko, Journal of Propulsion and Power 26, 609-637 (2010).

2. J. Sinko, S. Scharring, H.-A. Eckel, H.-P. Röser, and A. Sasoh, "Measurement Issues in Pulsed Laser Propulsion," in $6^{\text {th }}$ International Symposium on Beamed Energy Propulsion, edited by C. Phipps, K. Komurasaki, and J. Sinko, AIP Conference Proceedings 1230, American Institute of Physics, Melville, New York, 2010, pp. 125-136.

3. S. Scharring, H.-A. Eckel, H.-P. Röser, J. Sinko, and A. Sasoh, "Laser Propulsion Standardization Issues," in International Symposium on High Power Laser Ablation 2010, edited by C. Phipps, AIP Conference Proceedings 1278, American Institute of Physics, Melville, New York, 2010, pp. 780788.

4. S. Scharring, J. Sinko, A. Sasoh, H.-A. Eckel, and H.-P. Röser, International Journal of Aerospace Innovations 3, 33-43 (2011).

5. S. Scharring, E. Wollenhaupt, H.-A. Eckel, and H.-P. Röser, "Flight Experiments on Energy Scaling for In-Space Laser Propulsion," in $6^{\text {th }}$ International Symposium on Beamed Energy Propulsion, edited by C. Phipps, K. Komurasaki, and J. Sinko, AIP Conference Proceedings 1230, American Institute of Physics, Melville, New York, 2010, pp. 326-337. 
6. W. Schall, E. Zeyfang, W. Riede, and W. Mayerhofer, Propulsion device and method of generating shock waves, Patent DE 10017343 C2 (2000), filed Apr. 7 2000(in German) and US 2002/0047673 A1, filed Apr. 62001.

7. W. Bohn, and W. Schall, "Laser Propulsion Activities in Germany," in $1^{\text {st }}$ International Symposium on Beamed Energy Propulsion, edited by A. Pakhomov, American Institute of Physics, Melville, New York, 2003, vol. 664 of AIP Conference Proceedings, pp. 79-91.

8. W. Schall, W. Bohn, H.-A. Eckel, W. Mayerhofer, W. Riede, and E. Zeyfang, "Lightcraft Experiments in Germany," in Proceedings of High Power Laser Ablation III, edited by C. Phipps, 2000, vol. 4065 of Proceedings of SPIE, pp. 472-481.

9. S. Scharring, D. Hoffmann, H.-A. Eckel, and H.-P. Röser, Acta Astronautica 65, 1599-1615 (2009).

10. L. Myrabo, M. Libeau, E. Meloney, and R. Bracken, Pulsed laser propulsion performance of 11-cm parabolic 'bell' engines within the atmosphere, AIAA paper 2002-2206 (2002).

11. W. Bohn, "Laser Propulsion - Quo vadis," in $5^{\text {th }}$ International Symposium on Beamed Energy Propulsion, edited by A. Pakhomov, American Institute of Physics, Melville, New York, 2008, vol. 997 of AIP Conference Proceedings, pp. 47-55.

12. J. Sinko, S. Scharring, N. Ogita, A. Sasoh, H.-A. Eckel, and H.-P. Röser, "Update on $\mathrm{CO}_{2}$ Laser Ablation of Polyoxymethylene at $101 \mathrm{kPa}$," in International Symposium on High Power Laser Ablation 2010, edited by C. Phipps, AIP Conference Proceedings 1278, American Institute of Physics, Melville, New York, 2010, pp. 548-557.

13. C. Phipps, T. Turner, R. Harrison, G. York, W. Osborne, G. Anderson, X. Corlis, L. Haynes, H. Steele, K. Spicochi, and T. King, J. Appl. Phys. 64, 1083-1096 (1988).

14. J. Sinko, and C. Phipps, Appl. Phys. Lett. 95, 131105 (2009).

15. J. Sinko, and D. Gregory, "Models For Laser Ablation Mass Removal And Impulse Generation In Vacuum," in $6^{\text {th }}$ International Symposium on Beamed Energy Propulsion, edited by A. Pakhomov, American Institute of Physics, Melville, New York, 2010, vol. 1230 of AIP Conference Proceedings, pp. 193-203.

16. C. Phipps, R. Harrison, T. Shimada, G. York, T. Turner, X. Corlis, H. Steele, L. Haynes, and T. King, Laser and Particle Beams 8, 281-298 (1990).

17. J. Sinko, and D. Gregory, "Critical Fluence Effects in Laser Propulsion," in $7^{\text {th }}$ Conference on High Power Laser Ablation, edited by C. Phipps, Proceedings of SPIE 7005, SPIE, Bellingham, WA, 2008, pp. 1Z1-1Z11.

18. J. Sinko, N. Dhote, J. Lassiter, and D. Gregory, "Conical Nozzles for Pulsed Laser Propulsion," in $7^{\text {th }}$ Conference on High Power Laser Ablation, edited by C. Phipps, Proceedings of SPIE 7005, SPIE, Bellingham, WA, 2008, pp. 2Q1-2Q10.

19. J. Sinko, Vaporization and Shock Wave Dynamics for Impulse Generation in Laser Propulsion, Ph.D. thesis, University of Alabama in Huntsville (2008).

20. Technical polymers - datasheets, Tech. rep., Ensinger GmbH, Anröchte, Germany (2010), (in German).

21. Y. Rezunkov, M. Egorov, S. Rebrov, E. Repina, and A. Safronov, "Fine-Adjustment Thruster For Space Vehicles," in $6^{\text {th }}$ International Symposium on Beamed Energy Propulsion, edited by A. Pakhomov, American Institute of Physics, Melville, New York, 2010, vol. 1230 of AIP Conference Proceedings, pp. 309-318.

22. J. Sinko, C. Phipps, Y. Tsukiyama, N. Ogita, A. Sasoh, N. Umehara, and D. Gregory, "Critical Fluences and Modeling Of $\mathrm{CO}_{2}$ Laser Ablation Of Polyoxymethylene From Vaporization To The Plasma Regime," in $6^{\text {th }}$ International Symposium on Beamed Energy Propulsion, edited by A. Pakhomov, American Institute of Physics, Melville, New York, 2010, vol. 1230 of AIP Conference Proceedings, pp. 395-407.

23. J. Sinko, and A. Sasoh, "Survey Of $\mathrm{CO}_{2}$ Laser Ablation Propulsion With Polyoxymethylene Propellant," in $6^{\text {th }}$ International Symposium on Beamed Energy Propulsion, edited by A. Pakhomov, American Institute of Physics, Melville, New York, 2010, vol. 1230 of AIP Conference Proceedings, pp. 231-242.

24. A. Ageichik, E. Repina, Y. Rezunkov, and A. Safronov, Tech. Phys. 54, 402-409 (2009). 